\title{
Concomitant PI3K-AKT and p53 alterations in endometrial carcinomas are associated with poor prognosis
}

\author{
Lluis Catasus, Alberto Gallardo, Miriam Cuatrecasas and Jaime Prat \\ Department of Pathology, Hospital de la Santa Creu i Sant Pau, Autonomous University of Barcelona, \\ Barcelona, Spain
}

\begin{abstract}
The status of $\mathrm{p} 53$ and the phosphatidylinositol 3-kinase-AKT (PI3K-AKT) signaling pathway was investigated in 132 endometrial carcinomas, including endometrioid endometrial carcinomas, non-endometrioid endometrial carcinomas, and mixed endometrioid adenocarcinomas-non-endometrioid adenocarcinomas. Results were compared with the clinicopathologic parameters associated with prognosis, patients' follow-up, and other genetic alterations found frequently in these tumors. Molecular genetic differences between low-grade and high-grade endometrioid adenocarcinomas were encountered; ie, PIK3CA mutations were detected in 26 and $34 \%$ of cases, respectively. We found $p 53$ alterations in only $17 \%$ of high-grade endometrioid adenocarcinomas. In contrast, non-endometrioid adenocarcinomas had a higher frequency of p53 alterations (54\%), PIK3CA mRNA overexpression (45\%), and exon 20 PIK3CA mutations (21\%). In the mixed endometrioid adenocarcinomas-non-endometrioid adenocarcinomas, the most frequent alterations were p53 (50\%) and PIK3CA (44\%) mutations, followed by PTEN mutations (38\%). In some cases, $p 53$ and PIK3CA alterations coexisted, but they rarely coexisted with the PTEN mutations. Our findings suggest that the PIK3CA mutations are frequent events in endometrial carcinomas of any histological type. However, location of the PIK3CA mutations, either in exon 9 or exon 20, varies significantly according to the histologic grade and type of carcinoma. Carcinomas with exon 20 PIK3CA mutations or PIK3CA mRNA overexpression were often high-grade carcinomas associated with myometrial invasion; in contrast, tumors that carried exon 9 mutations were more likely to be low-grade carcinomas. The Kaplan-Meier analysis suggested that p53 alterations (strong immunoexpression or mutations) conferred a worse prognosis $(P=0.000)$. Although alterations in the PI3K-AKT signaling pathway alone did not influence overall survival, patients with deregulated PI3K-AKT pathway (PIK3CA and/or PTEN alterations) and $p 53$ alterations had shorter survival $(P=0.000)$ than patients with only $p 53$ alterations. Such a relationship was lost when we considered exon 9 PIK3CA mutations. Our results contribute to further characterize the molecular genetic model for endometrial carcinogenesis.

Modern Pathology (2009) 22, 522-529; doi:10.1038/modpathol.2009.5; published online 20 February 2009
\end{abstract}

Keywords: endometrial carcinoma; PIK3CA; p53; prognosis

Endometrial carcinoma is the most common malignant tumor of the female genital tract and the fourth most common female cancer in the Western world. ${ }^{1}$ Two main types have been recognized: ${ }^{2}$ type I are endometrioid adenocarcinomas, which represent $\sim 80 \%$ of endometrial carcinomas. ${ }^{3}$ They develop in peri- or post-menopausal women and are related to estrogen stimulation. They are predominantly low-grade endometrioid adenocarcinomas, often confined to the uterus and are frequently preceded by endometrial

Correspondence: Professor J Prat, MD, FRCPath, Hospital de la Santa Creu i Sant Pau, Department of Pathology, Avda. Sant Antoni M. Claret 167, 08025 Barcelona, Spain.

E-mail: jprat@santpau.cat

Received 17 October 2008; revised and accepted 20 January 2009; published online 20 February 2009 hyperplasia. Endometrioid adenocarcinomas frequently have microsatellite instability ${ }^{4}$ and mutations of the PTEN, ${ }^{5}$ PIK3CA, ${ }^{6} \mathrm{~K}$-Ras, ${ }^{7}$ and $\beta$-catenin genes. ${ }^{8,9}$ In contrast, type II tumors are high-grade nonendometrioid carcinomas which invade deeply into the myometrium and follow an aggressive clinical course. They occur in older women and are unrelated to estrogen stimulation. Non-endometrioid carcinomas are frequently associated with p53 mutations and chromosomal instability. ${ }^{10}$ Although this dualistic model shows some overlap, the clinical and pathologic differences are often paralleled by specific genetic alterations. ${ }^{4,11}$

The PI3K-AKT oncogenic signaling pathway is activated in many human epithelial cancers. ${ }^{12-15}$ Its activation counteracts directly the action of the lipid phosphatase PTEN, a negative regulator 
of PI3K-AKT. Activated PI3K-AKT regulates the expression of several downstream target genes that inhibit apoptosis and promote cell proliferation. The p110a catalytic subunit of PI3K (PIK3CA) is an oncogene located on chromosome $3 q 26.3$ and is mutated in several cancer types. ${ }^{12}$ The PIK3CA mutations increase PI3K kinase activity, cell survival, motility, and cell cycle progression. Mutations are usually missense and cluster in exons 9 (helical domain) and 20 (kinase domain). We have investigated recently the PIK3CA status in a large series of endometrioid adenocarcinomas and compared the results with the clinical and pathologic parameters associated with prognosis. ${ }^{16}$ In our series, the PIK $3 C A$ mutations occurred in $29 \%$ of endometrioid adenocarcinomas, all with myometrial invasion. We also found that histologic type, grade, and depth of myometrial invasion varied significantly depending on the location of PIK3CA mutations, either in exon 9 or exon $20 .^{16}$

$P T E N$ is a tumor-suppressor gene located in chromosome 10q23.3, a genomic region undergoing loss of heterozygosity in a wide variety of human cancers. PTEN encodes a phosphatase that antagonizes the PI3K-AKT pathway by dephosphorylating PIP3, a product of PI3K. In an earlier investigation, we found somatic PTEN mutations in $51-54 \%$ of endometrial carcinomas and they were far more frequent in endometrioid adenocarcinomas than in non-endometrioid adenocarcinomas. ${ }^{5,16}$

P53 mutations are the most characteristic genetic alterations of non-endometrioid adenocarcinomas. $P 53$ is a tumor-suppressor gene located in 17p13.1 that encodes p53 protein, a transcription factor that induces the expression of genes necessary for cell cycle arrest and apoptosis in response to DNA damage. Moreover, P53 inactivation is observed in over $50 \%$ of all human tumors. Immunohistochemical overexpression of p53 is found in most non-endometrioid adenocarcinomas (71-85\%) and may be useful in their distinction from endometrioid adenocarcinomas. ${ }^{17,18}$

Recently, important interactions between the PI3K-AKT and p53 signaling pathways have been described. $^{19-21}$ Studies on cell line suggest that activation of the PI3K pathway through PTEN or PIK3CA mutations causes activation of $p 53$. It has been reported that activation of the PI3K-AKT pathway together with $p 53$ inactivation results in malignant transformation. ${ }^{22,23}$ In this study, we have attempted to determine the status of the PI3K-AKT and p53 pathways in endometrial adenocarcinomas and compare the results with clinical and pathologic prognostic parameters and patients' follow-up.

\section{Materials and methods}

\section{Tissue Samples and DNA Isolation}

Samples from 132 endometrial carcinomas were retrieved from the Tumor Bank and the Surgical Pathology files of Hospital de la Santa Creu i Sant
Pau, Barcelona, Spain. All cases were reviewed and classified using the World Health Organization (WHO) criteria. Most of the cases (102 endometrioid adenocarcinomas) have been the subject of an earlier investigation. ${ }^{16}$ In this study, 30 high-grade carcinomas (14 non-endometrioid adenocarcinomas and 16 mixed endometrioid adenocarcinomas-non-endometrioid adenocarcinomas) were included. Genomic DNA from tumors and the corresponding non-tumor tissues was extracted using standard methods from frozen biopsies. All cases were anonymized and this study was approved by the Institutional Ethics Committee.

\section{PIK3CA, P53, and PTEN Mutational Analysis}

The PIK3CA, p53, and PTEN gene mutations were assessed on tumor DNA using polymerase chain reaction (PCR) amplification and subsequent sequencing analysis. Mutational analysis was performed using earlier-reported PCR conditions and primers for exons 9 and 20 of $P I K 3 C A,{ }^{12}$ exons 5-8 of p53, ${ }^{18}$ and exons $1-9$ of PTEN ${ }^{5}$ genes. The thermal cycling conditions included an initial $12 \mathrm{~min}$ at $94{ }^{\circ} \mathrm{C}$, followed by 40 cycles of $45 \mathrm{~s}$ at $94{ }^{\circ} \mathrm{C}, 45 \mathrm{~s}$ at specific annealing primer temperature of $52-62^{\circ} \mathrm{C}$, $1 \mathrm{~min}$ at $72^{\circ} \mathrm{C}$, and a final extension of $10 \mathrm{~min}$ at $72{ }^{\circ} \mathrm{C}$. The PCR conditions for exon 9 of PIK $3 C A$ were optimized to avoid mispriming with the PIK3CA pseudogene spanning exons 9-13 on chromosome 22. The PCR products were purified using the exoSAP-IT (USB, Cleveland, OH, USA) and subjected to direct sequencing using the ABI PRISM $^{\mathrm{TM}}$ big Dye terminator v1.1 cycle sequencing Kit (Applied Biosystems, Foster City, CA, USA). Sequencing fragments were detected by capillary electrophoresis using an automated ABI PRISM 310 Genetic Analyzer (Applied Biosystems).

\section{PIK3CA Gene Expression}

The mRNA levels of PIK3CA were evaluated by semiquantitative RT-PCR. cDNA was synthesized from $1 \mu \mathrm{g}$ of total RNA using the HighCapacity cDNA Reverse Transcription kit (Applied Biosystems). Subsequently, products were amplified with the 7300 Real Time PCR System (Applied Biosystems) using specific primers and fluorescent TaqMan probes. The expression levels were measured in triplicate. Normal endometrium pool tissue was used as a calibrator for quantitative Real Time PCR, and $A B L-1$ housekeeping gene was the reference for normalization. PIK3CA amplification was arbitrarily designated as two times or higher when compared with the endogenous control loci.

\section{Immunohistochemical Analysis}

Immunohistochemistry was performed on paraffinembedded tissue sections using the EnVision system 
(Dako) and diaminobenzidine as the chromogen. The reactions were carried out in a Dako Envision immunostainer. Tissue arrays were performed for an immunohistochemical analysis of pAkt, stathmin, ER, PR, $\beta$-catenin, p53, hMLH1, and hMSH2.

\section{Statistical Analysis}

Statistical analysis was performed using the statistical package SPSS/win 15.0 (SPSS, Chicago, IL, USA). The following clinicopathologic parameters were evaluated: age, tumor size, histologic type and grade, depth of myometrial invasion, lymphovascular invasion, clinicopathologic stage, hormone receptor status, p53 protein expression, PIK3CA, PTEN, and p53 mutations, as well as patients' outcome. A value of $P \leqslant 0.05$ was considered statistically significant. Overall survival was calculated from the date of diagnosis according to the Kaplan and Meier method. Multivariate Cox regression analysis was used to investigate the relationship between prognostic parameters and survival. The following parameters were introduced: PI3KAKT and $p 53$ alterations, histologic grade, stage, and vascular invasion.

\section{Results}

\section{Clinical and Pathologic Findings}

Patients' age ranged from 35 to 88 years (mean: 66.6 years). Tumor size varied from 0.4 to $11 \mathrm{~cm}$ (mean: $4.2 \mathrm{~cm})$. Of the 132 cases, $102(77 \%)$ were endometrioid adenocarcinomas, $14(11 \%)$ non-endometrioid adenocarcinomas, and $16(12 \%)$ mixed endometrioid adenocarcinomas-non-endometrioid adenocarcinomas. Of the 14 non-endometrioid adenocarcinomas, 6 were serous carcinomas, 4 clear cell carcinomas, and 4 mixed clear cell carcinomasserous carcinomas. Of the 16 mixed endometrioid adenocarcinomas-non-endometrioid adenocarcinomas, 9 were endometrioid adenocarcinomas-clear cell carcinomas and 7 endometrioid adenocarcinomas-serous carcinomas. Thirty-four tumors were grade $1(26 \%), 41$ grade $2(31 \%)$, and 57 grade 3 (43\%). Most tumors were FIGO stage I (89; 67\%), 16 (12\%) stage II, 20 (15\%) stage III, and 7 (5\%) stage IV. Myometrial invasion was found in 119 cases. It involved $\leqslant 1 / 2$ of the myometrial thickness in 64 cases and $>1 / 2$ in 55 . Thirty tumors $(23 \%)$ had lymphovascular invasion.

\section{Follow-up}

Follow-up information was obtained in 126 cases $(95.5 \%): 103(78 \%)$ patients were alive without clinical evidence of tumor at a mean follow-up interval of 3.68 years (range: 2 months-10 years); tumor persisted or recurred in $5(4 \%)$ patients at a mean follow-up interval of 1.9 years; 14 (11\%) patients died of tumor between 9 months and 8.4 years (mean: 2.2 years) postoperatively; and 4 (3\%) patients died of unrelated causes. Eleven patients had received tamoxifen for breast carcinoma. $\mathrm{Pa}-$ tients with low-stage tumors, who lacked vascular invasion, had significantly better survival $(P=0.001)$. Patients with non-endometrioid carcinomas had shorter survival than those with endometrioid carcinomas. In fact, 93\% (91/98) of patients with endometrioid adenocarcinomas survived, whereas only $39 \%(11 / 28)$ with non-endometrioid adenocarcinomas were alive. The difference was statistically significant $(P=0.000)$.

\section{PIK3CA Mutations and Gene Expression}

All endometrioid adenocarcinomas (102 cases) have been the subject of an earlier study, in which the PIK3CA mutations were identified in 19 of $73(26 \%)$ low-grade (grades 1 and 2) endometrioid adenocarcinomas and in 10 of 29 (34\%) high-grade (grade 3) endometrioid adenocarcinomas. ${ }^{16}$ Of the 30 highgrade carcinomas included in this study, the PIK3CA mutations were found in $21 \%(3 / 14)$ nonendometrioid adenocarcinomas and in 44\% (7/16) mixed endometrioid adenocarcinomas-non-endometrioid adenocarcinomas. All the PIK3CA mutations found in non-endometrioid adenocarcinomas and in mixed carcinomas occurred in exon 20 (Figure 1). The PIK3CA mutations were present in $4 / 13(31 \%)$ serous carcinomas and in $6 / 13(46 \%)$ clear cell carcinomas. The distribution of PIK3CA mutations is shown in Figure 2.

Overall 95\% of mutated tumors showed myometrial invasion. Histologic type and grade varied significantly according to the distribution of the PIK3CA mutations between exons 9 and 20 $(P=0.017$ and $P=0.003$, respectively). Mutations in exon 9 were found more frequently in low-grade (grades 1 and 2 endometrioid adenocarcinomas; $13 / 17 ; 76 \%$ ) than in high-grade carcinomas (grade 3 endometrioid adenocarcinomas, non-endometrioid

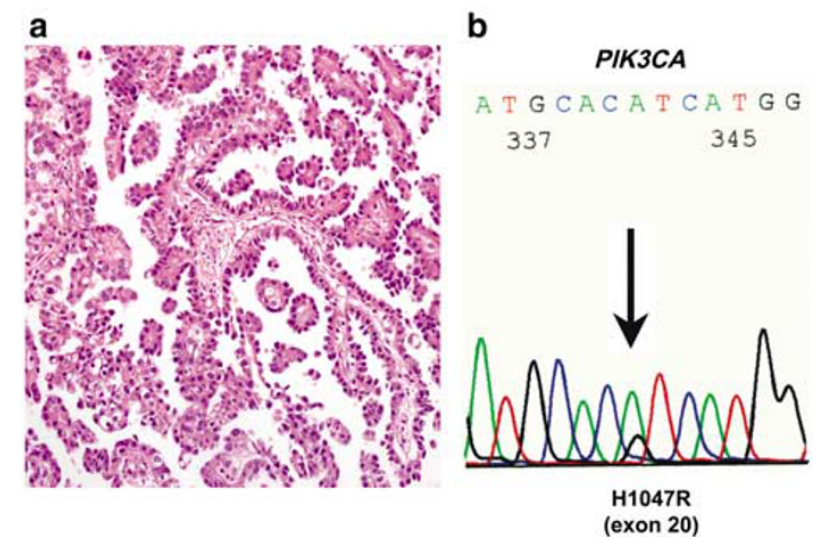

Figure 1 (a) Clear cell carcinoma. (b) Sequence analysis of exon 20 of PIK3CA reveals a missense mutation (H1047R). 


\begin{tabular}{lcc|} 
& \\
& & \\
Low-grade EEC & $13(65 \%)$ & $7(35 \%)$ \\
High-grade EEC & $4(33 \%)$ & $8(67 \%)$ \\
NEEC & $0(0 \%)$ & $3(100 \%)$ \\
Mixed EEC-NEEC & $0(0 \%)$ & $7(100 \%)$ \\
\hline Total & 17 & 25
\end{tabular}

Figure 2 Distribution of PIK3CA mutations in endometrial carcinomas. ABD, adaptor-binding domain; RBD, Ras-binding domain; EEC, endometrioid endometrial carcinoma; NEEC, nonendometrioid endometrial carcinoma.

adenocarcinomas, and mixed endometrioid adenocarcinomas-non-endometrioid adenocarcinomas; 4/17; $24 \%$ ). In contrast, exon 20 mutations were more common in high-grade $(18 / 25 ; 72 \%$ ) than in low-grade carcinomas $(7 / 25 ; 28 \%)$. Although mutated tumors had a higher frequency of lymphovascular invasion $(12 / 39 ; 31 \%)$ than non-mutated tumors $(18 / 93 ; 19 \%)$, the difference was not statistically significant $(P=0.153)$.

Twenty-two endometrial carcinomas were evaluated for the PIK3CA gene expression using real-time quantitative RT-PCR. We detected a two times or greater increase in the PIK3CA mRNA expression in 8 of $22(36 \%)$ adenocarcinomas compared with that in the normal control tissue. The PIK3CA expression was higher in non-endometrioid adenocarcinomas than in endometrioid adenocarcinomas and mixed carcinomas. No relationship was found between the PIK3CA overexpression and the clinical or pathologic features.

\section{P53 Alterations}

P53 alterations (strong immunoexpression or mutations) occurred more frequently in non-endometrioid adenocarcinomas $(7 / 13 ; 54 \%)$ and mixed endometrioid adenocarcinomas-non-endometrioid adenocarcinomas $(8 / 16 ; 50 \%)$ than in low-grade endometrioid adenocarcinomas $(1 / 60 ; 2 \%)$ or in high-grade endometrioid adenocarcinomas (5/29; $17 \%)(P=0.000)$ (Figure 3). p53 alterations were found in $7 / 12(58 \%)$ serous carcinomas and in $5 / 12$ $(42 \%)$ clear cell carcinomas, regardless of whether they were pure or mixed with an endometrioid adenocarcinomas component. In mixed serous-clear cell carcinomas, the p53 alterations were found in $3 / 4(75 \%)$ of cases. Tumors with $p 53$ alterations had lymphovascular invasion more frequently (8/21; $38 \%)$ than tumors with wild-type p53 (18/97; $18.5 \%)$. However, the difference did not reach statistical significance $(P=0.078)$. The P53 mutations in exons 5-8 were associated with strong p53 immunostaining in $65 \%$ of cases. There was a coexistence of the PIK3CA mutations and p53 alterations in $7 \%$ of cases (8/118), almost all of them were pure endometrioid adenocarcinomas or

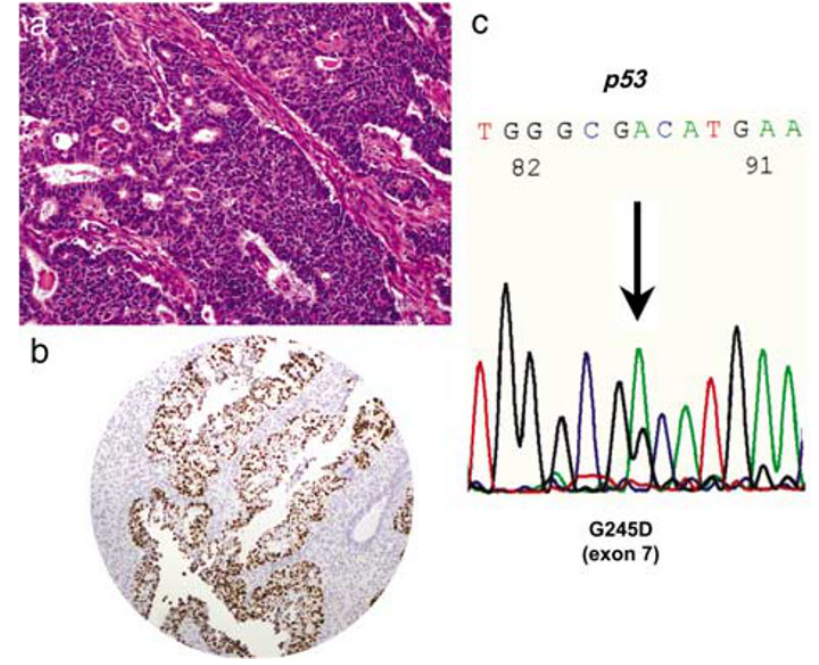

Figure 3 (a) Grade 3 endometrioid carcinoma. (b) Strong p53 immunoreaction. (c) Sequence analysis of p53 missense mutation in exon 7 (G245D).

mixed carcinomas with an endometrioid component.

\section{PTEN Mutations}

Mutations of PTEN had been analyzed earlier in 102 endometrioid adenocarcinomas. ${ }^{16}$ The molecular genetic analysis was expanded with 30 additional high-grade tumors (non-endometrioid adenocarcinomas and mixed endometrioid adenocarcinomasnon-endometrioid adenocarcinomas). The PTEN mutations were more common in pure endometrioid adenocarcinomas compared with that in non-endometrioid adenocarcinomas and mixed endometrioid adenocarcinomas-non-endometrioid adenocarcinomas. Results of the PIK3CA and PTEN mutations and of the p53 alterations are summarized in Table 1.

\section{Correlations of PIK3CA, PTEN, and P53 Alterations with Survival}

The prognostic significance of PIK3CA, PTEN, and p53 alterations was analyzed using the KaplanMeier method. Patients with p53 alterations (strong immunoexpression and/or mutations) had a shorter survival than those without them $(P=0.000)$ (Figure 4a). In contrast, no correlation with survival was encountered in carcinomas with or without mutations in the PIK3CA or PTEN genes only (Figure $4 \mathrm{~b}$ ). Moreover, the survival of patients with or without the PIK3-AKT pathway alterations (PIK3CA and/or PTEN mutations) was compared with p53 status (Figure 4c and d). Patients with concomitant p53 and PI3K-AKT alterations had shorter survival than patients with p53 alterations alone $(P=0.000)$. For this analysis, exon 9 PIK3CA mutations were excluded. In a multivariate analysis, PI3K-AKT and p53 concomitant alterations and vascular 
invasion were independent predictors of survival ( $P=0.000$ and $P=0.007$, respectively).

\section{Discussion}

We have carried out a comparative clinicopathologic and molecular genetic study of 132 endometrial carcinomas, including 102 endometrioid adenocarcinomas reported earlier, ${ }^{16}$ and 30 additional highgrade non-endometrioid adenocarcinomas and

Table 1 Molecular genetic alterations found in 132 endometrial carcinomas

\begin{tabular}{lccccc}
\hline & $\begin{array}{c}\text { EEC } \\
\text { low-grade }\end{array}$ & $\begin{array}{c}\text { EEC } \\
\text { high-grade }\end{array}$ & NEEC & $\begin{array}{c}\text { Mix } \\
\text { EEC-NEEC }\end{array}$ & Total \\
\hline PTEN & $55 \%$ & $59 \%$ & $0 \%$ & $38 \%$ & $48 \%$ \\
PIK3CA & $26 \%$ & $34 \%$ & $21 \%$ & $44 \%$ & $29.5 \%$ \\
P53 & $2 \%$ & $17 \%$ & $54 \%$ & $50 \%$ & $16 \%$ \\
\hline
\end{tabular}

EEC, endometrioid endometrial carcinoma; NEEC, non-endometrioid endometrial carcinoma; Mix, mixed. mixed carcinomas. Our study focused on the status of the PI3K-AKT signaling pathway and p53.

The reported frequency of the PIK3CA mutations in endometrial carcinomas ranges from 24 to $39 \% .^{6,15,16,24}$ However, none of the series investigated included non-endometrioid adenocarcinomas. To the best of our knowledge, our study is the first to describe PIK3CA mutations in non-endometrioid adenocarcinomas. We found $29.5 \%$ (39/132) PIK3$C A$ mutations in endometrial carcinomas including all histologic types. Mutations were more frequent in mixed endometrioid adenocarcinomas-non-endometrioid adenocarcinomas (44\%) than in pure endometrioid adenocarcinomas $(28 \%)$ or pure nonendometrioid adenocarcinomas $(21 \%)$. It should be noted that, type and histologic grade of the tumors varied significantly according to location of the PIK3CA mutations, either in exon 9 (helical domain) or in exon 20 (kinase domain). As reported earlier, the frequency of exon 9 mutations was higher in low-grade endometrioid adenocarcinomas than in high-grade endometrioid adenocarcinomas. Non-endometrioid adenocarcinomas and mixed a

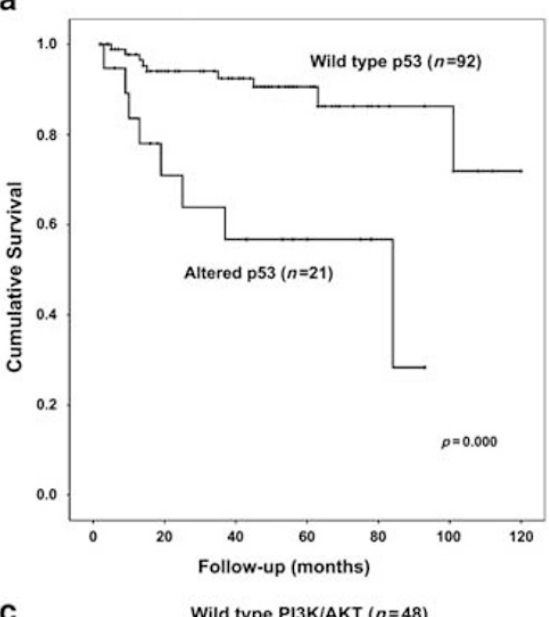

C

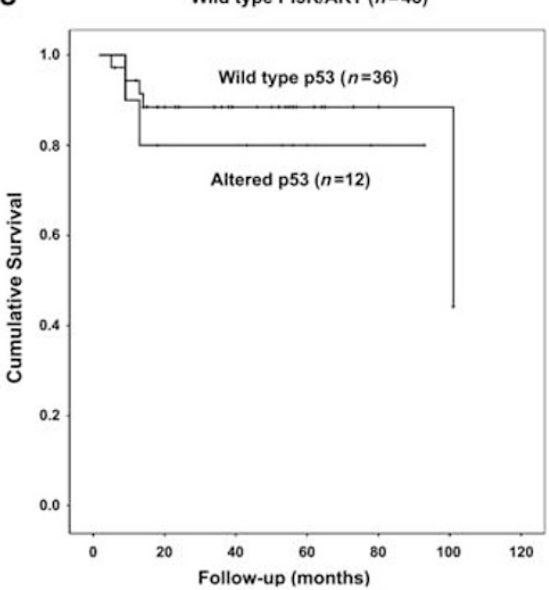

b

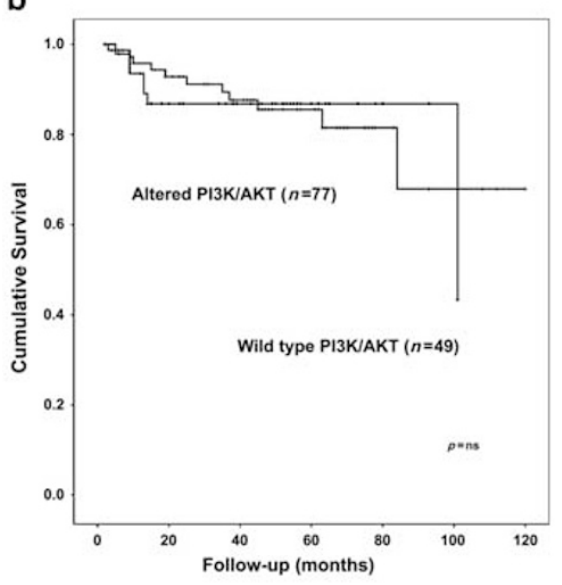

d Altered PI3K/AKT $(n=65)$

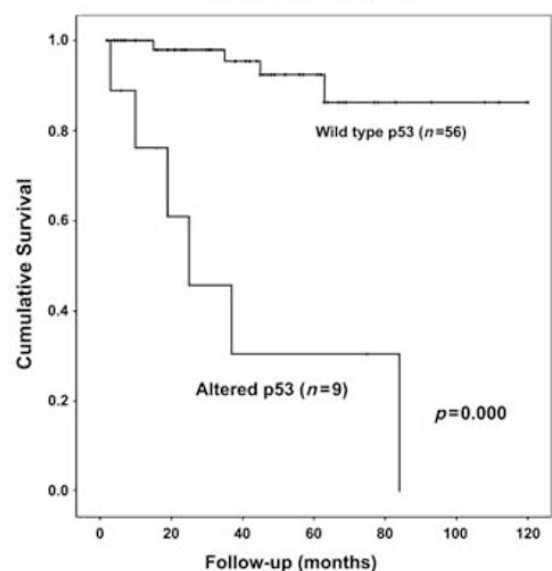

Figure 4 The Kaplan-Meier survival curves in endometrial carcinomas. (a) Patients with and without p53 alterations $(P=0.000)$. (b) Patients with and without PI3K-AKT pathway alterations $(P=\mathrm{ns})$. (c and d) Patients with and without PI3K-AKT and p53 alterations $(P=0.000)$. 
carcinomas exhibited exon 20 mutations exclusively. We also found that the PIK3CA mRNA expression was increased in non-endometrioid adenocarcinomas compared with that in endometrioid adenocarcinomas, mixed carcinomas, and normal tissues. Almost all tumors with exon 20 PIK3CA mutations and/or PIK3CA mRNA overexpression had myometrial invasion and tended to show lymphovascular invasion. Our findings provide further evidence that endometrial adenocarcinomas with mutations in the kinase domain (exon $20)$ or the PIK3CA mRNA overexpression are associated with adverse prognostic parameters. Although similar findings have been reported in high-grade ovarian carcinomas, ${ }^{25}$ reports have been contradictory regarding the prognostic significance of the PIK3CA helical and kinase domain mutations in breast carcinomas. ${ }^{26-29}$ However, it has been reported that chickens injected with exon 20 PIK3CA mutants developed larger tumors than those injected with exon 9 PIK3CA mutants. ${ }^{30}$

The distinct associations of the different PIK3CA mutations with histologic type, grade, and depth of myometrial invasion in endometrial carcinomas is consistent with the idea that different categories of mutants, defined by their structural and functional domains, would increase PI3K function by diverse mechanisms. Mutations in the kinase domain of exon 20 alter the catalytic loop and increase the specific activity of the enzyme, thus enhancing pathway activation. In contrast, mutations in the helical domain (exon 9) cluster on an exposed surface patch of the protein and may change its ability to interact with other regulatory proteins, which may be different for each tissue. ${ }^{31-33}$

As expected, analyses of the PTEN mutations revealed that these alterations are more common in pure endometrioid adenocarcinomas compared with that in non-endometrioid adenocarcinomas and mixed tumors. Different studies of colorectal and breast carcinomas have claimed that the PIK3CA and PTEN mutations are mutually exclusive and suggest that carcinogenic signaling through this pathway occurs either through the activation of PIK3CA or inactivation of PTEN. ${ }^{34,35}$ However, in endometrial carcinoma, coexistence of the PIK3CA and PTEN mutations ranges from 15 to $27 \%$ and the association is not statistically significant. ${ }^{6,15,16,24}$ In our series, the PTEN mutations were detected in lowand high-grade endometrioid adenocarcinomas and in mixed carcinomas with an endometrioid component, but were not found in non-endometrioid adenocarcinomas. The PTEN and PIK3CA mutations coexisted in $15 \%(20 / 132)$ of low- and high-grade endometrioid adenocarcinomas, and in one case of mixed endometrioid adenocarcinomas-non-endometrioid adenocarcinomas. Our results confirm that in endometrial carcinoma, the PIK3CA mutations coexist frequently with other alterations of genes upstream of the PI3K-AKT pathway, such as PTEN or K-Ras. A recent study ${ }^{36}$ has reconfirmed that the
PIK3CA mutations occur almost exclusively in invasive tumors, whereas upstream mutations of the PI3K-AKT pathway (PTEN and K-Ras mutations) occur with equal frequency in early-and latestage tumors. This finding suggests that the PIK3CA mutations cooperate with these alterations in the malignant transformation.

In our study, p53 alterations (strong immunoexpression and/or mutations) were more frequent in non-endometrioid adenocarcinomas (54\%) and mixed endometrioid adenocarcinomas-non-endometrioid adenocarcinomas (50\%) than in high-grade endometrioid adenocarcinomas $(17 \%)$ or low-grade endometrioid adenocarcinomas $(2 \%)$. The PIK3CA mutations, almost all located in exon 20, coexisted with p53 alterations in high-grade endometrioid adenocarcinomas and mixed carcinomas more frequently than in non-endometrioid adenocarcinomas. However, the PIK3CA mRNA overexpression occurred concomitantly with p53 alterations exclusively in non-endometrioid adenocarcinomas. Moreover, the p53 alterations coexisted with PTEN mutations in only three cases $(4 \%)$. In a recent study of ovarian carcinomas, ${ }^{25}$ p53 mutations coexisted with PIK3CA amplification in $7 \%$ of serous carcinomas. However, in ovarian endometrioid carcinomas, no association between the PIK3CA alterations and p53 mutations was found.

Recent studies have shown important interactions between the PI3K-AKT and p53 signaling pathways. $^{19,20}$ A functional p53 binding site has been identified within the PTEN promoter region, suggesting that $p 53$ is able to regulate PTEN expression. Other interaction sites at HDM2 may enable PTEN to regulate p53 protein stability. Moreover, a physical association between p53 and PTEN proteins, which would protect p53 from degradation, has been described. These observations suggest that p53 and PTEN form a positive feedback loop, in which p53 induces PTEN expression and PTEN reduces p53 degradation. It has also been shown that p53 binds directly to the PIK3CA promoter region and inhibits its activity. ${ }^{21}$ Moreover, activation of the PI3K-AKT signaling pathway by inactivating the PTEN mutations or activating the PIK3CA mutations would cause the activation of p53. Thus, activation of the PI3K-AKT pathway together with p53 inactivation would promote malignant transformation..$^{22,23}$

Our results suggest that activation of PI3K-AKT and p53 inactivation may have a concomitant negative effect on prognosis in high-grade endometrial carcinomas. In fact, the Kaplan-Meier analysis indicates that, by itself, p53 alterations (strong immunoexpression and/or mutations) are associated with poor prognosis $(P=0.000)$. In contrast, no correlation with survival was encountered in carcinomas with alterations in the PIK3CA or PTEN genes exclusively. Thus, as suggested earlier, ${ }^{15}$ activated PI3K-AKT pathway alone is not a prognostic marker in endometrial carcinomas. In breast carcinoma, however, aberrant PTEN tumor-suppres- 
sor activity has been associated with poor prognosis. ${ }^{37}$ In our cases with p53 alterations, deregulation of the PI3K-AKT signaling pathway (PIK $3 C A$ and/or PTEN alterations) was associated with worse prognosis than that of cases with p53 alterations alone. We found lower survival rates in carcinomas with both $p 53$ and PIK3CA alterations (exon 20 mutations and/or mRNA overexpression) and/or PTEN mutations, than in cases with p53 alterations exclusively using the Kaplan-Meier and multivariate analysis $(P=0.000)$. This association was lost when exon 9 PIK $3 C A$ mutations were considered. All patients who died of tumors with alterations in both p53 and PIK3-AKT had non-endometrioid adenocarcinomas or mixed endometrioid adenocarcinomas-non-endometrioid adenocarcinomas. Our data suggest that PIK3-AKT and p53 alterations have a negative synergistic influence on prognosis in endometrial carcinomas.

In summary, our study confirms that activation of the PI3K-AKT signaling pathway alone is not an adverse prognostic factor in endometrial carcinomas. However, simultaneous alterations both in the PI3K-AKT and the p53 pathways have a negative effect on prognosis and are associated with lower survival. Such an association is lost when mutations in exon 9 of PIK3CA are included. As exons 9 and 20 encode different domains, it is reasonable to speculate that they may have different oncogenic potential. Thus, the different mechanisms by which the two categories of PIK3CA mutations induce a gain of function may explain their different impact on prognosis. The PIK3CA mutations are frequent events in all histologic types of endometrial adenocarcinomas. We found that tumors with exon 20 PIK3CA mutations or PIK3CA mRNA overexpression are often high-grade carcinomas associated with myometrial invasion compared with those with exon 9 mutations.

\section{Acknowledgement}

This work was supported by Grants FIS PI04-1891, PI06-0950, and RTICCCFIS RD06/0020/0015, Department of Health, Spain, Marato TV3 2005-47, and Fundación Mutua Madrileña-07.

\section{Conflict of interest}

The authors declare no conflict of interest.

\section{References}

1 American Cancer Society. Cancer Facts and Figures 2008. American Cancer Society: Atlanta, GA, 2008.

2 Bokhman JV. Two pathogenetic types of endometrial carcinoma. Gynecol Oncol 1983;15:10-17.
3 Prat J, Gallardo A, Cuatrecasas M, et al. Endometrial carcinoma: pathology and genetics. Pathology 2007;39:72-87.

4 Catasús L, Machin P, Matias-Guiu X, et al. Microsatellite instability in endometrial carcinomas clinicopathologic correlations in a series of 42 cases. Hum Pathol 1998;29:1160-1164.

5 Bussaglia E, del Rio E, Matias-Guiu X, et al. PTEN mutations in endometrial carcinomas: a molecular and clinicopathologic analysis of 38 cases. Hum Pathol 2000;31:312-317.

6 Velasco A, Bussaglia E, Pallares J, et al. PIK3CA gene mutations in endometrial carcinoma. Correlation with PTEN and K-Ras alterations. Hum Pathol 2006;37:1465-1472.

7 Lagarda H, Catasus L, Arguelles R, et al. K-ras mutations in endometrial carcinomas with microsatellite instability. J Pathol 2001;193:193-199.

8 Machin P, Catasus L, Pons C, et al. CTNNB1 mutations and beta-catenin expression in endometrial carcinomas. Hum Pathol 2002;33:206-212.

9 Moreno-Bueno G, Hardisson D, Prat J, et al. Abnormalities of the APC/beta-catenin pathway in endometrial cancer. Oncogene 2002;21:7981-7990.

10 Prat J, Oliva E, Lerma E, et al. Uterine papillary serous adenocarcinoma. A 10 case study of p53 and c-erbB 2 expression and DNA content. Cancer 1994;74:1778-1883.

11 Lax SF. Molecular genetic pathways in various types of endometrial carcinoma: from a phenotypical to a molecular-based classification. Virchows Arch 2004;444:213-223.

12 Samuels Y, Wang Z, Bardelli A, et al. High frequency of mutations of the PIK3CA gene in human cancers. Science 2004;304:554.

13 Campbell IG, Russell SE, Choong DY, et al. Mutation of the PIK3CA gene in ovarian and breast cancer. Cancer Res 2004;64:7678-7681.

14 Lee JW, Soung YH, Kim SY, et al. PIK3CA gene is frequently mutated in breast carcinomas and hepatocellular carcinomas. Oncogene 2005;24:1477-1480.

15 Oda K, Stokoe D, Taketani Y, et al. High frequency of coexistent mutations of PIK3CA and PTEN genes in endometrial carcinoma. Cancer Res 2005;65:10669-10673.

16 Catasus L, Gallardo A, Cuatrecasas M, et al. PIK3CA mutations in the kinase domain (exon 20) of uterine endometrial adenocarcinomas are associated with adverse prognostic parameters. Mod Pathol 2008;21:131-139.

17 Moll UM, Chalas E, Auguste M, et al. Uterine papillary serous carcinoma evolves via a p53-driven pathway. Hum Pathol 1996;27:1295-1300.

18 Tashiro $\mathrm{H}$, Isacson $\mathrm{C}$, Levine $\mathrm{R}$, et al. p53 gene mutations are common in uterine serous carcinoma and occur early in their pathogenesis. Am J Pathol 1997;150:177-185.

19 Stambolic V, MacPherson D, Sas D, et al. Regulation of PTEN transcription by p53. Mol Cell 2001;8:317-325.

20 Freeman DJ, Li AG, Wei G, et al. PTEN tumor suppressor regulates p53 protein levels and activity through phosphatase-dependent and -independent mechanisms. Cancer Cell 2003;3:117-130.

21 Astanehe A, Arenillas D, Wasserman WW, et al. Mechanisms underlying p53 regulation of PIK3CA transcription in ovarian surface epithelium and in ovarian cancer. J Cell Sci 2008;121:664-674.

22 Lee C, Kim JS, Waldman T. Activated PI3K signaling as an endogenous inducer of p53 in human cancer. Cell Cycle 2007;6:394-396. 
23 Kim JS, Lee C, Bonifant CL, et al. Activation of p53-dependent growth suppression in human cells by mutations in PTEN or PIK3CA. Mol Cell Biol 2007;27:662-677.

24 Hayes MP, Wang H, Espinal-Witter R, et al. PIK3CA and PTEN mutations in uterine endometrioid carcinoma and complex atypical hyperplasia. Clin Cancer Res 2006;12:5932-5935.

25 Willner J, Wurz K, Allison KH, et al. Alternate molecular genetic pathways in ovarian carcinomas of common histological types. Hum Pathol 2007;38:607-613.

26 Lai YL, Mau BL, Cheng WH, et al. PIK3CA exon 20 mutation is independently associated with a poor prognosis in breast cancer patients. Ann Surg Oncol 2008;15:1064-1069.

27 Lerma E, Catasus L, Gallardo A, et al. Exon 20 PIK3CA mutations decreases survival in aggressive (HER-2 positive) breast carcinomas. Virchows Arch 2008;453:133-139.

$28 \mathrm{Li} \mathrm{SY,} \mathrm{Rong} \mathrm{M,} \mathrm{Grieu} \mathrm{F,} \mathrm{et} \mathrm{al.} \mathrm{PIK3CA} \mathrm{mutations} \mathrm{in}$ breast cancer are associated with poor outcome. Breast Cancer Res Treat 2006;96:91-95.

29 Barbareschi M, Buttitta F, Felicioni L, et al. Different prognostic roles of mutations in the helical and kinase domains of the PIK3CA gene in breast carcinomas. Clin Cancer Res 2007;13:6064-6069.

30 Bader AG, Kang S, Vogt PK. Cancer-specific mutations in PIK3CA are oncogenic in vivo. Proc Natl Acad Sci USA 2006;103:1475-1479.
31 Gymnopoulos M, Elsliger M-A, Vogt PK. Rare cancerspecific mutations in PIK3CA show gain of function. Proc Natl Acad Sci USA 2007;104:5569-5574.

32 Huang CH, Mandelker D, Schmidt-Kittler O, et al. The structure of a human p110alpha/p85alpha complex elucidates the effects of oncogenic PI3Kalpha mutations. Science 2007;318:1744-1748.

33 Zhao L, Vogt PK. Helical domain and kinase domain mutations in p110alfa of phosphatidylinositol 3-kinase induce gain of function by different mechanisms. Proc Natl Acad Sci USA 2008;105:2652-2657.

34 Saal LH, Holm K, Maurer M, et al. PIK3CA mutations correlate with hormone receptors, node metastasis, and ERBB2, and are mutually exclusive with PTEN loss in human breast carcinoma. Cancer Res 2005;65:2554-2559.

35 Frattini M, Signoroni S, Pilotti S, et al. Phosphatase protein homologue to tensin expression and phosphatidylinositol-3 phosphate kinase mutations in colorectal cancer. Cancer Res 2005;65:11227.

36 Oda K, Okada J, Timmerman L, et al. PIK3CA cooperates with other phosphatidylinositol 3'-kinase pathway mutations to effect oncogenic transformation. Cancer Res 2008;68:8127-8136.

37 Saal LH, Johansson P, Holm K, et al. Poor prognosis in carcinoma is associated with a gene expression signature of aberrant PTEN tumor suppressor pathway activity. Proc Natl Acad Sci USA 2007;104: 7564-7569. 